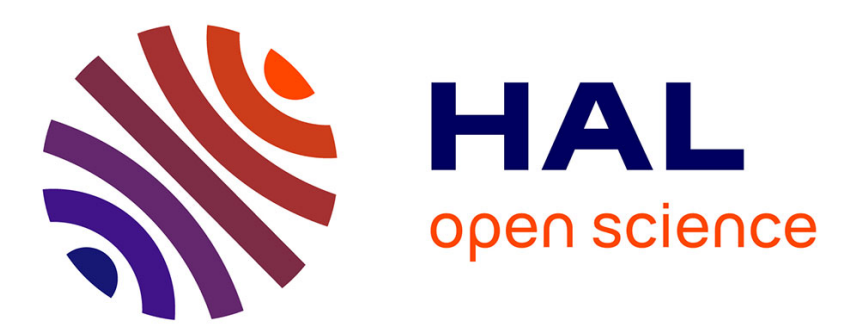

\title{
A boosting approach for prostate cancer detection using multi-parametric MRI
}

Guillaume Lemaître, Joan Massich, Robert Martí, Jordi Freixenet, Vilanova Joan C, Paul Walker, Désiré Sidibé, Fabrice Mériaudeau

\section{- To cite this version:}

Guillaume Lemaître, Joan Massich, Robert Martí, Jordi Freixenet, Vilanova Joan C, et al.. A boosting approach for prostate cancer detection using multi-parametric MRI. International Conference on Quality Control and Artificial Vision (QCAV) 2015, Jun 2015, Le Creusot, France. hal-01235890

\section{HAL Id: hal-01235890 \\ https://u-bourgogne.hal.science/hal-01235890}

Submitted on 4 Dec 2015

HAL is a multi-disciplinary open access archive for the deposit and dissemination of scientific research documents, whether they are published or not. The documents may come from teaching and research institutions in France or abroad, or from public or private research centers.
L'archive ouverte pluridisciplinaire HAL, est destinée au dépôt et à la diffusion de documents scientifiques de niveau recherche, publiés ou non, émanant des établissements d'enseignement et de recherche français ou étrangers, des laboratoires publics ou privés. 


\title{
A boosting approach for prostate cancer detection using multi-parametric MRI
}

\author{
Guillaume Lemaître ${ }^{a, b}$ and Joan Massich ${ }^{a}$ and Robert Martí ${ }^{b}$ and Jordi Freixenet ${ }^{b}$ and \\ Joan C. Vilanova ${ }^{c}$ and Paul M. Walker ${ }^{d}$ and Désiré D. Sidibé ${ }^{a}$ and Fabrice Mériaudeau ${ }^{a}$ \\ ${ }^{a}$ LE2I-UMR CNRS 6306, Université de Bourgogne, 12 rue de la Fonderie, 71200 Le Creusot, France; \\ ${ }^{b}$ ViCOROB, Universitat de Girona, Campus Montilivi, Edifici P4, 17071 Girona, Spain; \\ ${ }^{c}$ Department of Magnetic Resonance, Clinica Girona, Lorenzana 36, 17002 Girona, Spain

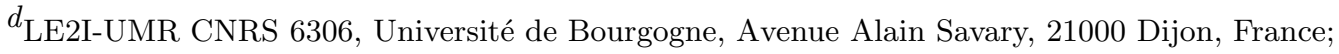

\begin{abstract}
Prostate cancer has been reported as the second most frequently diagnosed men cancers in the world. In the last decades, new imaging techniques based on MRI have been developed in order to improve the diagnosis task of radiologists. In practise, diagnosis can be affected by multiple factors reducing the chance to detect potential lesions. Computer-aided detection and computer-aided diagnosis have been designed to answer to these needs and provide help to radiologists in their daily duties. In this study, we proposed an automatic method to detect prostate cancer from a per voxel manner using 3T multi-parametric Magnetic Resonance Imaging (MRI) and a gradient boosting classifier. The best performances are obtained using all multi-parametric information as well as zonal information. The sensitivity and specificity obtained are 94.7\% and 93.0\%, respectively and an Area Under Curve (AUC) of 0.968.
\end{abstract}

Keywords: Gradient boosting, multi-parametric MRI, prostate cancer, computer-aided diagnosis

\section{INTRODUCTION}

On a worldwide scale, prostate cancer (PCa) has been reported as the second most frequently diagnosed men cancers accounting for $13.6 \%{ }^{1}$ Statistically, the estimated number of new diagnosed cases was 899,000 with no less than 258,100 estimated deaths. ${ }^{1}$ In United States, aside from skin cancer, PCa was declared to be the most commonly diagnosed cancer among men, implying that around one in seven men will be diagnosed with $\mathrm{PCa}$ during his lifetime. ${ }^{2}$

Since its introduction in mid-1980s, prostate-specific antigen (PSA) is widely used for PCa screening ${ }^{3}$ and has shown to improve early detection of $\mathrm{PCa} .{ }^{4}$ However, several trials conducted in Europe and United States conclude that PSA screening suffers from low specificity. ${ }^{5-7}$ Thus, current research focuses on developing new screening methods to improve PCa detection. In this perspective, Magnetic Resonance Imaging (MRI) techniques have recently shown promising results for PCa detection. Furthermore, three different modalities are currently investigated: (i) $\mathrm{T}_{2}$ Weighted ( $\mathrm{T}_{2}-\mathrm{W}$ ) MRI, (ii) Dynamic Contrast-Enhanced (DCE) MRI and (iii) Diffusion Weighted (DW) MRI.

Focusing on the state-of-the-art, ${ }^{8}$ it can be observed that extensive research has been carried out in order to investigate the contributions of machine learning classifiers for PCa detection using the three aforementioned 3T multi-parametric MRI such as Support Vector Machines (SVM),${ }^{9-13}$ probabilistic boosting tree ${ }^{14}$ or probabilistic neural network. ${ }^{14}$ However, these studies use different datasets and evaluation statistics to report their results. ${ }^{8}$ Thus, no fair comparisons between these different studies are possible. ${ }^{8}$

In this research, we investigate the performance of gradient boosting for $\mathrm{PCa}$ detection using 3T multiparametric MRI. Two different features extraction strategies have been chosen in order to feed the classifier: (i) voxel-based and (ii) 3D texton-based. An evaluation of both strategies as well as the contribution of each modality is provided. Furthermore, the dataset used for this experimentation is part of our future benchmarking platform I2CVB available at http://visor.udg. edu/i2cvb/ and is ready for future comparisons.

Further author information: (Send correspondence to G.L.) G.L.: E-mail: guillaume.lemaitre@udg.edu 


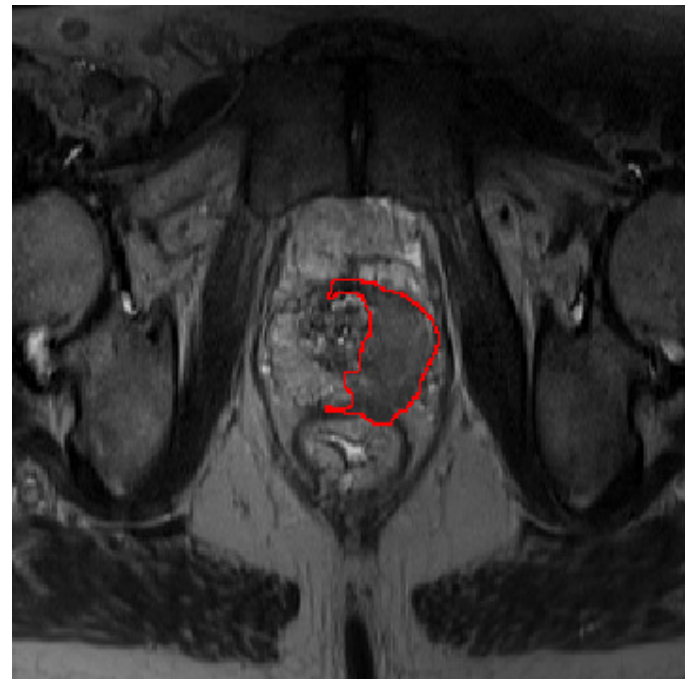

(a)

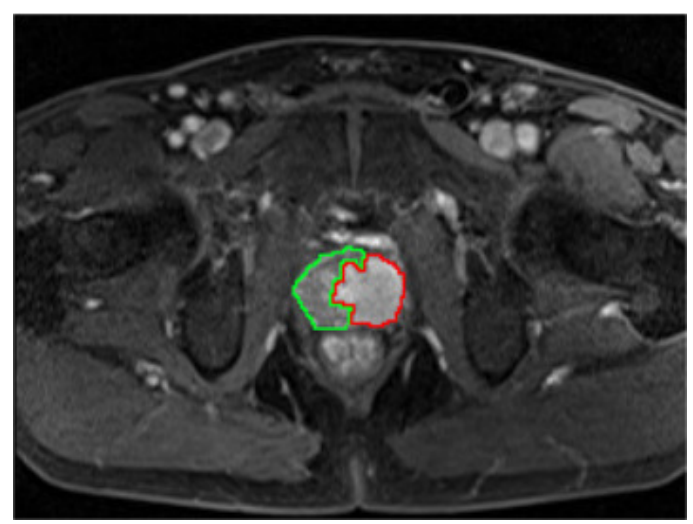

(c)

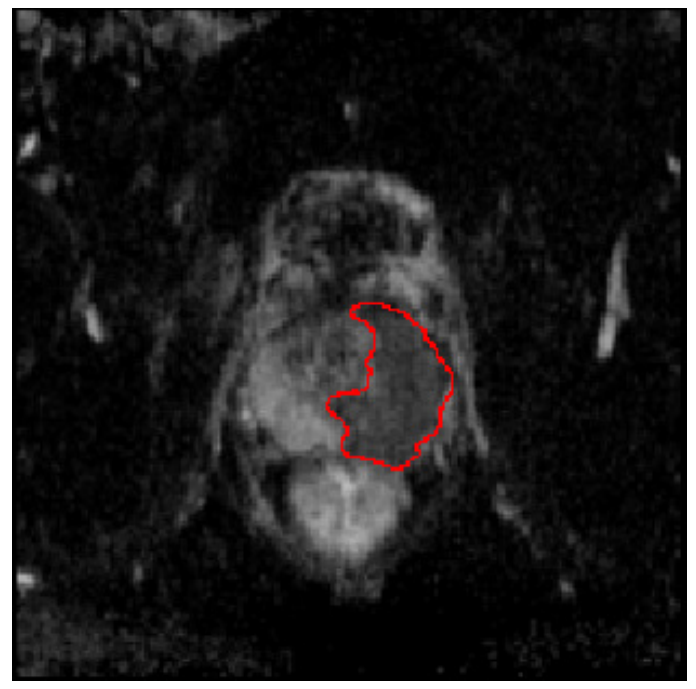

(b)

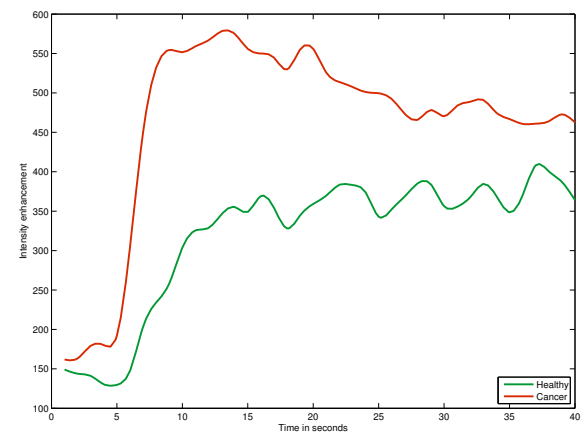

(d)

Figure 1: Multi-parametric MRI data acquired for our experiment. The cancer is highlighted in red in the different images: (a) T2-W-MRI modality, (b) ADC map, (c) and (d) DCE-MRI modality.

\section{MATERIAL AND METHODS}

\subsection{Data}

The multi-parametric MRI was acquired from a cohort of patients with higher-than-normal level of PSA. The acquisition was performed using a 3T whole body MRI scanner (Siemens Magnetom Trio TIM, Erlangen, Germany) using sequences to obtain $\mathrm{T}_{2}$-W MRI (see Fig. 1a), DCE MRI (see Fig. 1c and Fig. 1d) and DW MRI. Aside of the MRI examinations, these patients also underwent a guided-biopsy. Finally, the dataset was composed of a total of 20 patients of which 18 patients had biopsy proven PCa and 2 patients were "healthy" with negative biopsies. The prostate organ as well as the prostate zones (i.e., peripheral zone (PZ) and central gland $(\mathrm{CG})$ ) and PCa were manually segmented by an experienced radiologist. Therefore, 13 patients had a PCa in the PZ, 3 patients had PCa in the CG, 2 patients had invasive PCa in both PZ and CG and finally 2 patients were considered as "healthy".

The ADC (see Fig. 1b) maps were computed using the scanner software and the DW MRI. The DCE MRI sequence consists in a kinetic study composed of 40 samples over time. These DCE MRI sequences and ADC maps were resampled using the spatial information of the $\mathrm{T}_{2}$-W MRI sequence with dimensions of $448 \times 360 \times 64$ 
and voxel spacing of $0.68 \times 0.68 \times 1.25 \mathrm{~mm}^{3}$. Linear interpolation was used to compute missing data during the up-sampling. The resampling was implemented in $\mathrm{C}++$ using the Insight Segmentation and Registration Toolkit. ${ }^{15}$

Due to the large number of samples available at a voxel scale, the dataset was pre-processed in order to deal with a balanced dataset allowing to not bias the results. Therefore, all the positive samples (i.e., PCa voxels) were stored and an equal number of negative samples (i.e., "healthy" voxels) were randomly selected from the larger original pool. Thus, the total amount of positive and negative samples considered in our experiments accounted for 218,423 voxels.

\subsection{Classification framework}

\subsubsection{Feature extraction strategies}

Table 1: Overview of voxel features extracted in our classification framework.

\begin{tabular}{|c|c|c|c|}
\hline Extraction strategy & Name & Size & Short description \\
\hline \multirow{5}{*}{ Voxel-based } & $\mathrm{V}_{\mathrm{T}_{2}-\mathrm{W}}$ & 1 & Intensity of a voxel in the $\mathrm{T}_{2}$-W MRI \\
\hline & $\mathrm{V}_{\mathrm{ADC}}$ & 1 & Intensity of a voxel in the ADC map \\
\hline & $\mathrm{V}_{\mathrm{DCE}}$ & 40 & Intensities of a voxel along the whole serie in the DCE MRI \\
\hline & $\mathrm{V}_{\mathrm{PZ}}$ & 1 & Boolean value of a voxel membership to the $\mathrm{PZ}$ \\
\hline & $\mathrm{V}_{\mathrm{CG}}$ & 1 & Boolean value of a voxel membership to the $\mathrm{CG}$ \\
\hline \multirow{5}{*}{ 3D texton-based } & $\mathrm{T}_{\mathrm{T}_{2}-\mathrm{W}}$ & 243 & $\begin{array}{l}\text { Intensities vector for a window of } 9 \times 9 \times 3 \text { voxels in the } \\
\mathrm{T}_{2} \text {-W MRI }\end{array}$ \\
\hline & $\mathrm{T}_{\mathrm{ADC}}$ & 243 & $\begin{array}{l}\text { Intensities vector for a window of } 9 \times 9 \times 3 \text { voxels in the } \\
\text { ADC map }\end{array}$ \\
\hline & $\mathrm{T}_{\mathrm{DCE}}$ & 9720 & $\begin{array}{l}\text { Intensities vector for a window of } 9 \times 9 \times 3 \text { along the whole } \\
\text { serie in the DCE MRI }\end{array}$ \\
\hline & $\mathrm{T}_{\mathrm{PZ}}$ & 243 & $\begin{array}{l}\text { Boolean vector of voxels memberships to the } \mathrm{PZ} \text { for a win- } \\
\text { dow of } 9 \times 9 \times 3\end{array}$ \\
\hline & $\mathrm{T}_{\mathrm{CG}}$ & 243 & $\begin{array}{l}\text { Boolean vector of voxels memberships to the CG for a win- } \\
\text { dow of } 9 \times 9 \times 3\end{array}$ \\
\hline
\end{tabular}

A summary of the extracted features as well as the chosen strategies are summarized in Table 1. Two main strategies are applied to extract features. In the voxel-based approach, at each voxel location, the intensities for the different MRI modalities are extracted as well as the membership of this voxel to belong to the PZ or CG. The 3D texton-based approach extend this extraction for a 3D window of size $9 \times 9 \times 3$ around the central voxel. In both case, the vectors $V(\cdot)$ and $T(\cdot)$ extracted are scaled using min-max normalization.

Then, the different concatenation of the vectors $V(\cdot)$ and $T(\cdot)$ are summarized in Table 2. Different combinations are further tested in order to observe the contribution of each data feature.

\subsubsection{Gradient boosting}

In this research, a gradient boosting classifier ${ }^{16}$ originally proposed by Friedman ${ }^{17,18}$ is used for the implementation of our computer-aided detection and diangosis (CAD) system for PCa. Gradient boosting is in fact a reformulation of the well-known AdaBoost ${ }^{19}$ in which the problem of finding "boots" is tackled as a numerical optimization. In a greedy manner, a strong classifier is constructed by iteratively finding the best pair of real-valued weak learner function (e.g., regression trees) and its corresponding weight which minimize a given differentiable loss function. This minimization can be carried out via gradient descent or quadratic approximation. ${ }^{20}$ 
Table 2: Overview of the different combinations of features tested for the classification.

\begin{tabular}{|c|c|c|c|c|c|c|c|c|c|c|c|}
\hline Voxel-based & $\mathrm{V}_{\mathrm{T}_{2}-\mathrm{W}}$ & $\mathrm{V}_{\mathrm{ADC}}$ & $\mathrm{V}_{\mathrm{DCE}}$ & $\mathrm{V}_{\mathrm{PZ}}$ & $\mathrm{V}_{\mathrm{CG}}$ & Texton-based & $\mathrm{T}_{\mathrm{T}_{2}-\mathrm{W}}$ & $\mathrm{T}_{\mathrm{ADC}}$ & $\mathrm{T}_{\mathrm{DCE}}$ & $\mathrm{T}_{\mathrm{PZ}}$ & $\mathrm{T}_{\mathrm{CG}}$ \\
\hline 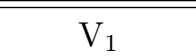 & $\bar{\Omega}$ & $\bar{x}$ & $\bar{x}$ & $\bar{x}$ & $\bar{x}$ & 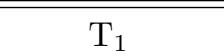 & $\bar{\Omega}$ & $\bar{x}$ & $\bar{x}$ & $\bar{x}$ & $\bar{x}$ \\
\hline $\mathrm{V}_{2}$ & $x$ & $\checkmark$ & $x$ & $x$ & $x$ & $\mathrm{~T}_{2}$ & $x$ & $\checkmark$ & $x$ & $x$ & $x$ \\
\hline $\mathrm{V}_{3}$ & $x$ & $x$ & $\checkmark$ & $x$ & $x$ & $\mathrm{~T}_{3}$ & $x$ & $x$ & $\checkmark$ & $x$ & $x$ \\
\hline $\mathrm{V}_{4}$ & $\checkmark$ & $\checkmark$ & $x$ & $x$ & $x$ & $\mathrm{~T}_{4}$ & $\checkmark$ & $\checkmark$ & $x$ & $x$ & $x$ \\
\hline $\mathrm{V}_{5}$ & $\checkmark$ & $x$ & $\checkmark$ & $x$ & $x$ & $\mathrm{~T}_{5}$ & $\checkmark$ & $x$ & $\checkmark$ & $x$ & $x$ \\
\hline $\mathrm{V}_{6}$ & $x$ & $\checkmark$ & $\checkmark$ & $x$ & $x$ & $\mathrm{~T}_{6}$ & $x$ & $\checkmark$ & $\checkmark$ & $x$ & $x$ \\
\hline $\mathrm{V}_{7}$ & $\checkmark$ & $\checkmark$ & $\checkmark$ & $x$ & $x$ & $\mathrm{~T}_{7}$ & $\checkmark$ & $\checkmark$ & $\checkmark$ & $x$ & $x$ \\
\hline $\mathrm{V}_{8}$ & $\checkmark$ & $\checkmark$ & $\checkmark$ & $\checkmark$ & $\checkmark$ & $\mathrm{T}_{8}$ & $\checkmark$ & $\checkmark$ & $\checkmark$ & $\checkmark$ & $\checkmark$ \\
\hline
\end{tabular}

The size of the feature vectors extracted for classification is problematic and it can lead to the non convergence of some well-known classification method such as SVM. ${ }^{21}$ Unlike SVM, boosting leads to consistent classification, even with early-stopping conditions. ${ }^{22}$ Furthermore, gradient boosting has been proved to outperform AdaBoost ${ }^{23}$ motivating our choice for this specific classifier. In this work, the weak learner function used is the regression trees while the loss function is an exponential loss.

\subsubsection{Validation model}

All the different combinations reported in Table 2 were classified using a ten-fold cross-validation procedure, of which nine-fold were used as training samples and one-fold was kept as testing samples and the experiments were repeated for ten iterations.

\section{RESULTS AND DISCUSSION}

The classification results obtained are given in terms of sensitivity and specificity and depicted in Fig. 2. Subsequently, further analysis in terms of Receiver Operating Characteristic (ROC) curves and Area Under Curve (AUC) is shown in Fig. 3.

The best classification performance is achieved using the 3D texton-based extraction strategy and a combination of the three different modalities and the zonal information. The sensitivity and specificity obtained are $94.7 \%$ and $93.0 \%$, respectively and an AUC of 0.986 .

Analyzing the classification outcomes of each single modality, the DCE MRI is the most discriminative feature with superior performance than the combination of $\mathrm{T}_{2}-\mathrm{W} \mathrm{MRI}$ and ADC map together. However, the two latter mentioned modalities provide relevant information since that the combination of the three of them enhances the reported sensitivity and specificity.

Integrating information about the prostate zones (i.e., PZ and CG) boosts the classification performance. More precisely, this feature allows to improve greatly the specificity and slightly the sensitivity.

In overall, the 3D texton-based strategy leads to better classification results while compared with the voxelbased strategy for all the mono and multi-parametric combinations experimented. Thus, integrating spatial information about the neighborhood of a given voxel leads to drastic improvements.

\section{CONCLUSION}

In this study, an exhaustive analysis of classification of 3T multi-parametric MRI data using a gradient boosting classifier has been carried out. The best classification performances are obtained by extracting the features using a 3D texton-based strategy and using the information from all the modalities as well as the zonal information. A maximum sensitivity and specificity of $86.9 \%$ and $84.6 \%$ respectively as well as an AUC of 0.935 , are reached. 


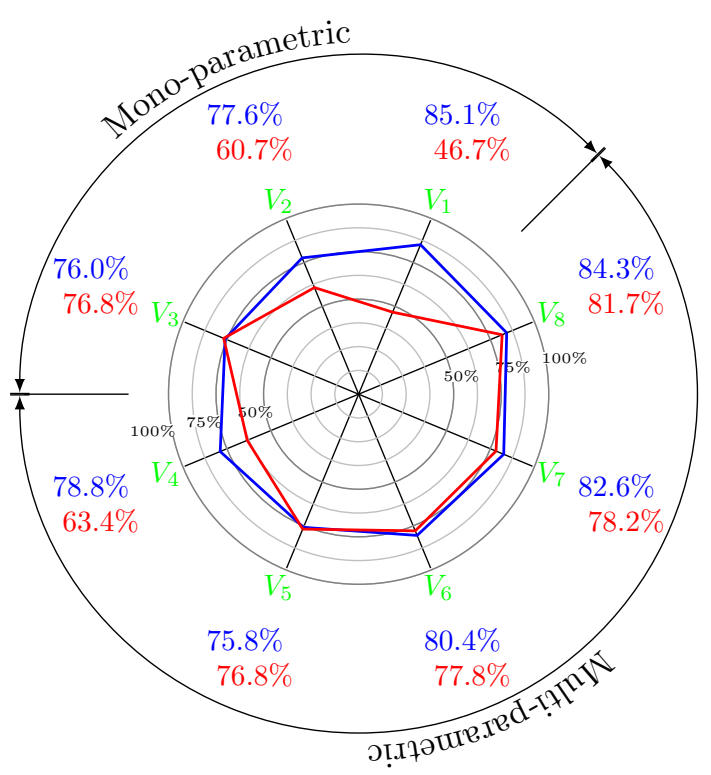

(a) Voxel-based features

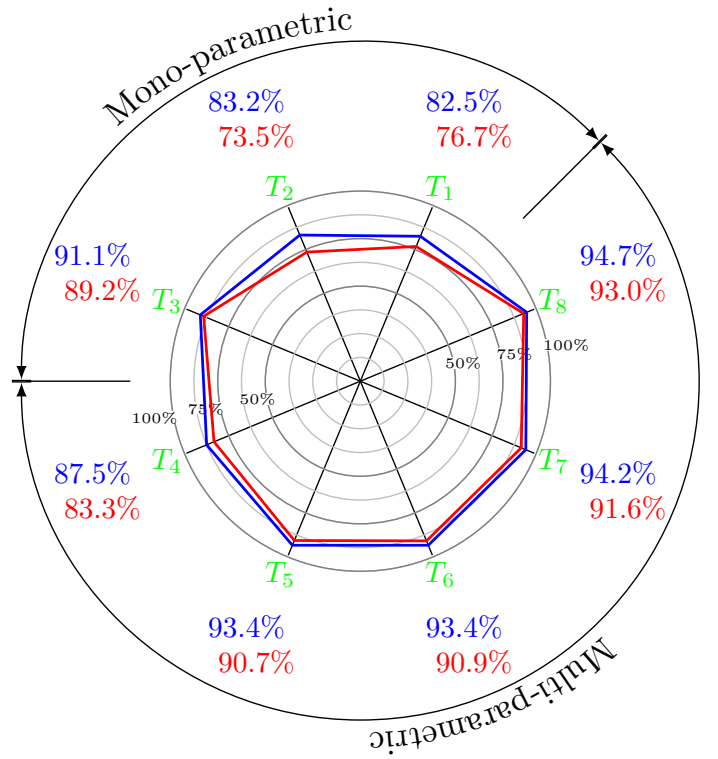

(b) 3D texton-based features

Figure 2: Graphical and numerical comparisons between the combination of features introduced in Table 2 in terms of sensitivity and specificity are illustrated in blue and red, respectively.

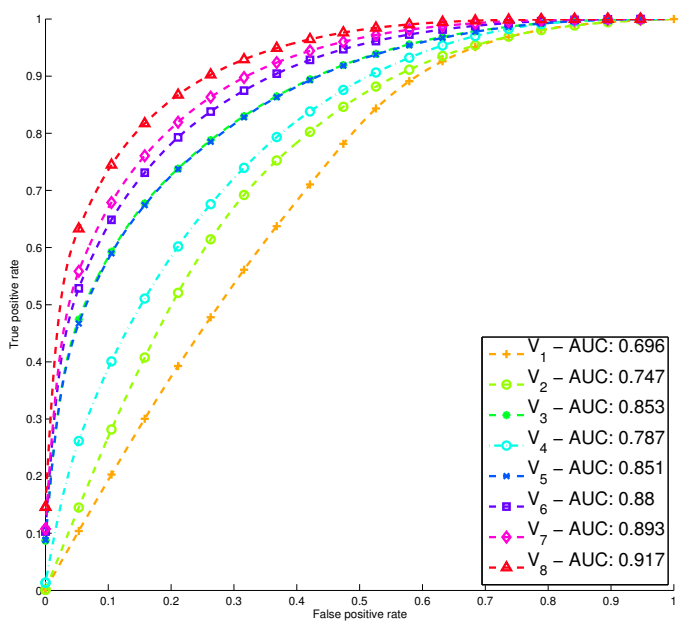

(a) ROC curves for voxel-based approach.

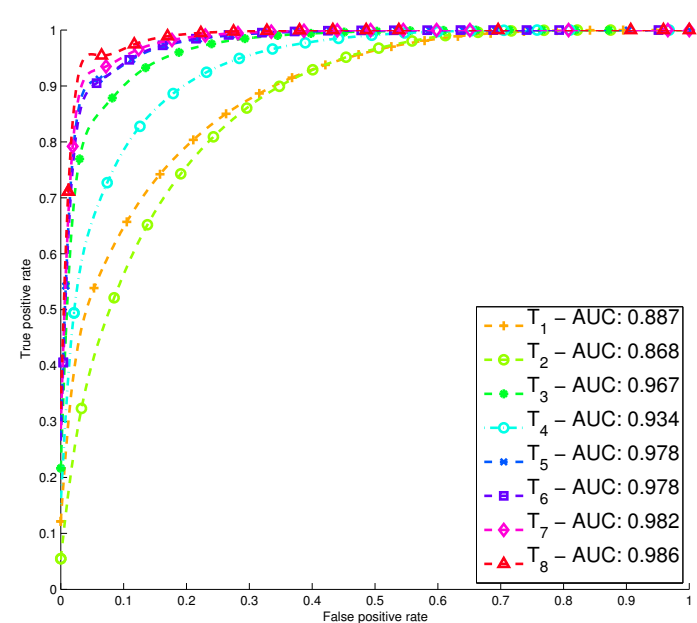

(b) ROC curves for 3D texton-based approach.

Figure 3: ROC curves for the both voxel-based and 3D texton-based approaches with different multi-parametric MRI combinations.

Two avenues for future research can be explored. First, the registration and segmentation of the multiparametric data ${ }^{24,25}$ has been discarded and could be performed ahead of the classification to study the possible improvements implied using in-house developed methods. ${ }^{26,27}$ Then, other features than intensities could be extracted and the results could be compared with the described experiments here, since the dataset used is public. 


\section{ACKNOWLEDGMENTS}

Guillaume Lemaitre was supported by the Generalitat de Catalunya (grant nb. FI-DGR2012) and partly by the Mediterranean Office for Youth (grant nb. 2011/018/06). We would like also to thank the Clinica Girona (Catalunya, Espanya) and the Centre Hospitalier of Dijon (France) for providing the MRI images used in this research.

\section{REFERENCES}

1. J. Ferlay, H. R. Shin, F. Bray, D. Forman, C. Mathers, and D. M. Parkin, "Estimates of worldwide burden of cancer in 2008: GLOBOCAN 2008," Int. J. Cancer 127, pp. 2893-2917, Dec 2010.

2. R. Siegel, J. Ma, Z. Zou, and A. Jemal, "Cancer statistics, 2014," CA: A Cancer Journal for Clinicians 64(1), pp. 9-29, 2014.

3. R. Etzioni, D. F. Penson, J. M. Legler, D. di Tommaso, R. Boer, P. H. Gann, and E. J. Feuer, "Overdiagnosis due to prostate-specific antigen screening: lessons from U.S. prostate cancer incidence trends," J. Natl. Cancer Inst. 94, pp. 981-990, Jul 2002.

4. R. Chou, J. M. Croswell, T. Dana, C. Bougatsos, I. Blazina, R. Fu, K. Gleitsmann, H. C. Koenig, C. Lam, A. Maltz, J. B. Rugge, and K. Lin, "Screening for prostate cancer: a review of the evidence for the U.S. Preventive Services Task Force," Ann. Intern. Med. 155, pp. 762-771, Dec 2011.

5. G. L. Andriole, E. D. Crawford, R. L. Grubb, S. S. Buys, D. Chia, T. R. Church, M. N. Fouad, E. P. Gelmann, P. A. Kvale, D. J. Reding, J. L. Weissfeld, L. A. Yokochi, B. O’Brien, J. D. Clapp, J. M. Rathmell, T. L. Riley, R. B. Hayes, B. S. Kramer, G. Izmirlian, A. B. Miller, P. F. Pinsky, P. C. Prorok, J. K. Gohagan, and C. D. Berg, "Mortality results from a randomized Prostate-cancer screening trial," New England Journal of Medicine 360(13), pp. 1310-1319, 2009.

6. J. Hugosson, S. Carlsson, G. Aus, S. Bergdahl, A. Khatami, P. Lodding, C. G. Pihl, J. Stranne, E. Holmberg, and H. Lilja, "Mortality results from the Göteborg randomised population-based prostate-cancer screening trial," Lancet Oncol. 11, pp. 725-732, Aug 2010.

7. F. H. Schröder, J. Hugosson, M. J. Roobol, T. L. Tammela, S. Ciatto, V. Nelen, M. Kwiatkowski, M. Lujan, H. Lilja, M. Zappa, L. J. Denis, F. Recker, A. Pez, L. Määttänen, C. H. Bangma, G. Aus, S. Carlsson, A. Villers, X. Rebillard, T. van der Kwast, P. M. Kujala, B. G. Blijenberg, U.-H. Stenman, A. Huber, K. Taari, M. Hakama, S. M. Moss, H. J. de Koning, and A. Auvinen, "Prostate-cancer mortality at 11 years of follow-up," New England Journal of Medicine 366(11), pp. 981-990, 2012.

8. G. Lemaître, R. Martí, J. Freixenet, J. C. Vilanova, P. M. Walker, and F. Meriaudeau, "Computer-Aided Detection and Diagnosis for prostate cancer based on mono and multi-parametric MRI: A Review " Computers in Biology and Medicine (0), pp. -, 2015.

9. G. J. S. Litjens, P. C. Vos, J. O. Barentsz, N. Karssemeijer, and H. J. Huisman, "Automatic computer aided detection of abnormalities in multi-parametric prostate MRI," in Proc. SPIE 7963, Medical Imaging 2011: Computer-Aided Diagnosis, pp. 79630T-79630T-7, 2011.

10. G. Litjens, O. Debats, W. van de Ven, N. Karssemeijer, and H. Huisman, "A pattern recognition approach to zonal segmentation of the prostate on MRI," Med Image Comput Comput Assist Interv 15(Pt 2), pp. 413420, 2012.

11. G. Litjens, O. Debats, J. Barentsz, N. Karssemeijer, and H. Huisman, "Computer-aided detection of prostate cancer in MRI," Medical Imaging, IEEE Transactions on 33, pp. 1083-1092, May 2014.

12. P. Liu, S. Wang, B. Turkbey, P. C. P. Grant, K.and Pinto, B. J. Wood, and R. M. Summers, "A prostate cancer computer-aided diagnosis system using multimodal magnetic resonance imaging and targeted biopsy labels," in Proc. SPIE 8670, Medical Imaging 2013: Computer-Aided Diagnosis, pp. 86701G-86701G-6, 2013.

13. Y. Peng, Y. Jiang, C. Yang, J. Brown, T. Antic, I. Sethi, C. Schmid-Tannwald, M. Giger, S. Eggener, and A. Oto, "Quantitative analysis of multiparametric prostate MR images: differentiation between prostate cancer and normal tissue and correlation with Gleason score-a computer-aided diagnosis development study," Radiology 267, pp. 787-796, June 2013. 
14. S. Viswanath, B. N. Bloch, J. Chappelow, P. Patel, N. Rofsky, R. Lenkinski, E. Genega, and A. Madabhushi, "Enhanced multi-protocol analysis via intelligent supervised embedding (EMPrAvISE): detecting prostate cancer on multi-parametric MRI," in Proc. SPIE 7963, Medical Imaging 2011: Computer-Aided Diagnosis, 2011.

15. H. J. Johnson, M. McCormick, L. Ibáñez, and T. I. S. Consortium, The ITK Software Guide. Kitware, Inc., third ed., 2013. In press.

16. C. Becker, R. Rigamonti, V. Lepetit, and P. Fua, "Supervised feature learning for curvilinear structure segmentation," in Medical Image Computing and Computer-Assisted Intervention MICCAI 2013, K. Mori, I. Sakuma, Y. Sato, C. Barillot, and N. Navab, eds., Lecture Notes in Computer Science 8149, pp. 526-533, Springer Berlin Heidelberg, 2013.

17. J. H. Friedman, "Stochastic Gradient Boosting," Computational Statistics and Data Analysis 38, pp. 367378, 1999.

18. J. H. Friedman, "Greedy Function Approximation: A Gradient Boosting Machine," Annals of Statistics 29, pp. 1189-1232, 2000.

19. Y. Freund and R. Schapire, "A decision-theoretic generalization of on-line learning and an application to boosting," Journal of Computer and System Sciences 55(1), pp. 119 - 139, 1997.

20. Z. Zheng, H. Zha, T. Zhang, O. Chapelle, K. Chen, and G. Sun, "A general boosting method and its application to learning ranking functions for web search neur," in Inf. Proc. Sys. Conf, pp. 1697-1704, 2008.

21. I. Chan, W. Wells, R. V. Mulkern, S. Haker, J. Zhang, K. H. Zou, S. E. Maier, and C. M. Tempany, "Detection of prostate cancer by integration of line-scan diffusion, T2-mapping and T2-weighted magnetic resonance imaging; a multichannel statistical classifier," Med Phys 30, pp. 2390-2398, Sep 2003.

22. T. Zhang and B. Yu, "Boosting with early stopping: Convergence and consistency," Ann. Statist. 33, pp. 1538-1579, 082005.

23. R. Caruana and A. Niculescu-Mizil, "An empirical comparison of supervised learning algorithms," in Proceedings of the 23rd International Conference on Machine Learning, ICML '06, pp. 161-168, ACM, (New York, NY, USA), 2006.

24. S. Ghose, A. Oliver, R. Marti, X. Llado, J. C. Vilanova, J. Freixenet, J. Mitra, D. Sidibe, and F. Meriaudeau, "A survey of prostate segmentation methodologies in ultrasound, magnetic resonance and computed tomography images," Comput Methods Programs Biomed 108, pp. 262-287, Oct 2012.

25. B. Zitová and J. Flusser, "Image registration methods: a survey," Image and Vision Computing 21(11), pp. $977-1000,2003$.

26. S. Ghose, A. Oliver, J. Mitra, R. Mart, X. Llad, J. Freixenet, D. Sidib, J. C. Vilanova, J. Comet, and F. Meriaudeau, "A supervised learning framework of statistical shape and probability priors for automatic prostate segmentation in ultrasound images," Medical Image Analysis 17(6), pp. 587 - 600, 2013.

27. J. Mitra, Z. Kato, R. Marti, A. Oliver, X. Llado, D. Sidibe, S. Ghose, J. C. Vilanova, J. Comet, and F. Meriaudeau, "A spline-based non-linear diffeomorphism for multimodal prostate registration," Med Image Anal 16, pp. 1259-1279, Aug 2012. 